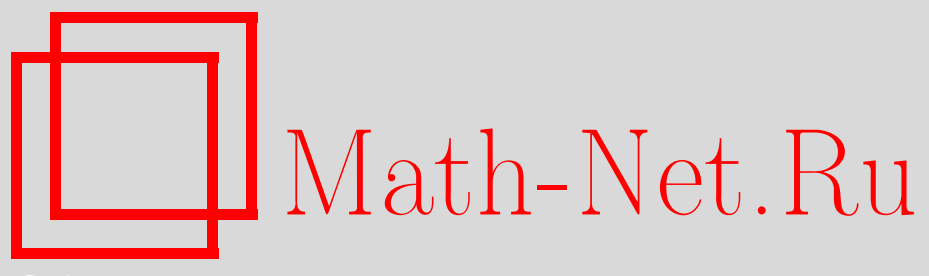

Ю. Л. Ершов, О. А. Клименко, И. И. Матвеева, В. В. Пикалов, Математическая информационная система MathTree, УМH, 2007, том 62, выпуск 5, 133-142

DOI: https://doi.org/10.4213/rm8524

Использование Общероссийского математического портала Math-Net.Ru подразумевает, что вы прочитали и согласны с пользовательским соглашением http://www.mathnet.ru/rus/agreement

Параметры загрузки:

IP : 3.89 .197 .203

26 апреля 2023 г., 16:02:35

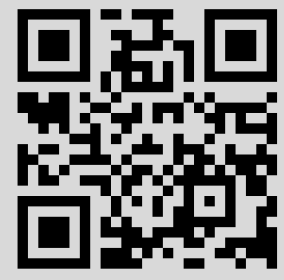




\title{
Математическая информационная система MathTree
}

\author{
Ю. Л. Ершов, О. А. Клименко, И. И. Матвеева, В. В. Пикалов \\ В статье дается описание математической информационной системы \\ MathTree, разработанной и развиваемой в Сибирском отделении Россий- \\ ской академии наук. Освещаются возможности, современное состояние и \\ пути развития представленной системы.
}

Библиография: 23 названия.

СОДЕРЖАНИЕ

1. Введение................................................. 133

2. Необходимость создания ................................. 134

3. Предлагаемая информация................................ 134

4. Возможности системы .................................. 138

5. Современное состояние системы ............................ 140

Список цитируемых Интернет-ресурсов и литературы ............... 141

\section{1. Введение}

Настоящая статья посвящена математической информационной системе MathTree [1], разработка которой была начата в 2003 году в рамках интеграционного проекта Сибирского отделения Российской академии наук "Разработка древовидного каталога математических Интернет-ресурсов". Цель проекта состояла в создании информационной системы для упорядочивания существующих математических ресурсов и предоставления дополнительных возможностей для поиска нужной информации. Первая версия системы MathTreе появилась в 2004 году [2]. В настоящее время работы по наполнению и совершенствованию системы продолжаются в рамках междисциплинарного интеграционного проекта СО РАН "Древовидный каталог математических интернет-ресурсов".

Система MathTreе предназначена для российских и зарубежных ученых, которые используют математический аппарат, а также для преподавателей, аспирантов и студентов. Информация представлена на русском и английском языках.

(С Ю. Л. Ершов, О.А. Клименко, И. И. МАтвеЕвА, В. В. ПиКАЛов, 2007 


\section{2. Необходимость создания}

Всем хорошо известен афоризм $Ф$. Бэкона: "Кто владеет информацией владеет миром", и поэтому проблема поиска нужной информации всегда будет актуальной. Современные информационные технологии открывают большие возможности по поиску и обмену информацией, что помогает справиться с такой проблемой, как ее труднодоступность. Однако, не успев решить эту проблему, люди столкнулись с другой: "Как в этом все возрастающем потоке информации найти нужную?" На решение этой проблемы было направлено создание поисковых систем, таких как Google, Yahoo, Rambler, Яндекс и т. д. Но, как известно, универсальный не значит лучший, и если пользователя интересует конкретная область знания, то он предпочтет обратиться к информационной системе, посвященной этой области, чем "выуживать" нужную информацию из "паутины", называемой Интернетом. Однако тематические информационные системы сейчас находятся на стадии становления, и математика не является исключением. Существующие математические информационные ресурсы разрознены и слабо систематизированы, причем в большей степени это касается русскоязычных ресурсов [3]. Поэтому необходимость создания системы MathTree была обусловлена проблемой упорядочивания существующих и вновь создаваемых математических ресурсов, и в первую очередь русскоязычных.

Цель настоящей статьи не состоит в проведении сравнительного анализа между системой MathTree и существующими информационными системами по математике. Каждый заинтересованный читатель может сделать это самостоятельно, выбрав наиболее удобные для его целей системы или отдельные математические ресурсы. При их поиске пользователь может обратиться к упомянутым выше поисковым системам, воспользоваться дополнительным специализированным сервисом этих систем (например, Google Scholar [4] для поиска статей с полной информацией о цитировании) или использовать коллекции математических электронных ресурсов, которые представлены на страницах математических сообществ (см., например, [5]-[8]), университетов (см., например, [9], [10]) и библиотек (см., например, [11], [12]). В настоящее время существует ряд российских математических информационных систем, среди которых следует особо отметить общероссийский математический портал Math-Net.Ru [13], созданный и развиваемый Математическим институтом им. В. А. Стеклова РАН совместно с Отделением математических наук РАН. Наша цель - рассказать о недавно созданной и постоянно развивающейся системе MathTree, которая пока мало известна широкому кругу российских и зарубежных ученых, но которая дополняет существующие системы, предоставляя новые возможности.

\section{3. Предлагаемая информация}

При разработке структуры системы MathTree в основу была положена систематизация ресурсов по типам и математическим направлениям.

Вначале мы остановимся на систематизации по типам, поскольку этот принцип сортировки используется в большинстве существующих информационных систем. В настоящее время ресурсы в MathTree упорядочены по следующим 
типам: журналы, конференции, персоны, публикации, библиотеки, организации, научные сообщества, программное обеспечение, научные школы, семинары, другое.

Категории "жсурналь”, “конферениии”, “персонь” являются самыми распространенными типами систематизации ресурсов по областям знаний, и, как правило, они представлены в каждой подобной системе. К категории "публикации" относятся ресурсы, посвященные отдельной книге, статье, учебному пособию и т. д. В том случае, когда ресурс посвящен большой коллекции публикаций или собранию книг, он систематизируется по типу "библиотека". K категории "организации" относятся ресурсы, касающиеся организаций математического профиля, отделов, факультетов, лабораторий, кафедр, т. е. научных или образовательных подразделений. Естественным дополнением этой категории является категория "научные сообщества", она была введена для систематизации ресурсов, посвященных определенной тематике. Как правило, эти ресурсы поддерживаются специалистами в соответствующей области, которые собирают информацию, связанную с направлением своих исследований. К этой категории, например, относятся общество по линейной алгебре, общество по разностным уравнениям. В настоящее время в каждой области математики существует специализированное программное обеспечение, разработанное крупными коллективами, и пакеты программ, созданные отдельными учеными, поэтому в системе MathTree соответствующие ресурсы отнесены к категории "программное обеспечение".

Следует особо отметить категорию “научные школь”. Русская, советская и российская наука знаменита своими научными школами, в том числе математическими, которые воспитали плеяду выдающихся ученых. С момента выхода постановления правительства от 23 мая 1996 года о поддержке ведущих научных школ Российской Федерации был создан ряд ресурсов, посвященных той или иной научной школе; эти ресурсы составили основу категории "научнъе школь" в MathTree. K сожалению, не у каждой школы в настоящее время имеется электронный ресурс, но продолжение программы по государственной поддержке научных школ позволяет надеяться, что количество таких ресурсов будет увеличиваться. Наличие таких ресурсов с информацией на русском и английском языках будет способствовать более широкому освещению традиций и достижений отечественной науки.

В последнее время в MathTree был введен новый тип ресурсов "семинары". Необходимость его создания была обусловлена, во-первых, тем, что эти ресурсы практически не представлены в других подобных системах, а во-вторых, интересом к этому типу ресурсов со стороны пользователей, в первую очередь студентов, аспирантов и соискателей.

Математические ресурсы многообразны, и не каждый можно отнести к тому или иному существующему типу. В этом случае в системе MathTree pecypc заносится в категорию "другое". В настоящее время в этой категории представлены, например, ресурсы по открытым математическим проблемам, ресурсы, посвященные диссертационным советам, и др. При большом количестве ресурсов, отвечающих определенному типу, в системе MathTree предусмотрена возможность введения новых категорий.

Как мы отмечали выше, сортировка по перечисленным типам (или их части) традиционно используется в существующих информационных системах. Одна- 
ко особенностью тематических информационных систем является принадлежность ресурсов не только к определенному типу, но и направлению. Зачастую пользователю нужна специализированная информация. Например, каждого ученого интересуют журналы и конференции не только общематематической направленности, но и по его тематике. Если исследователю необходимо применение численных методов, то он предпочтет обратиться к программному обеспечению, разработанному для его круга задач. При упорядочивании ресурсов только по типам без учета направления пользователь сталкивается с проблемой самостоятельного поиска в большом количестве информации и выбора нужной, что неизбежно приводит к потере времени. Поэтому, наряду с систематизацией ресурсов по перечисленным выше типам, в MathTree ресурсы упорядочены по направлениям математики. В настоящее время выделены следующие семь направлений:

- алгебра и логика;

- анализ;

- геометрия и топология;

- дифференциальные, интегральные и разностные уравнения;

- теория вероятностей и математическая статистика;

- математическое программирование и Computer Science;

- приложения.

Внутри каждого указанного раздела имеются дополнительные подразделы, отвечающие более специализированному направлению. При классификации и создании подразделов за основу был взят математический рубрикатор MSC (Mathematics Subject Classification), используемый в настоящее время в большинстве математических журналов, а также в реферативных журналах Маthematical Reviews и Zentralblatt MATH. Степень специализации по каждому направлению своя и зависит от количества ресурсов. Например, раздел "Алгебра и логика" содержит 4 основных подраздела: алгебра; логика; теория чисел; универсальная алгебра. Каждый из этих разделов, в свою очередь, также содержит дополнительные подразделы, при этом степень специализации достигает 4 уровней. Раздел “Дифференциальные, интегральные и разностные уравнения" содержит 5 основных подразделов: обыкновенные дифференциальные и разностные уравнения; уравнения с частными производными; динамические системы и эргодическая теория; интегральные уравнения; обратные задачи; при этом степень специализации достигает 5 уровней. По направлению "Математическое программирование и Computer Science" степень специализации достигает 8 уровней. Чтобы сделать предлагаемую специализацию по направлениям математики более наглядной, в системе MathTreе пользователю предлагается также древовидное представление, где каждая ветвь отвечает соответствующему подразделу. На каждом уровне в каждом подразделе ресурсы упорядочены по типам, указанным выше.

Предлагаемая в MathTree систематизация по типам и направлениям позволяет находить очень специализированную информацию. Так, например, в подразделе "Дифференциальные, интегральные и разностные уравнения / Обратные задачи / Приложения обратных задач / Вычислительная томография" широко представлены ресурсы по различным направлениям медицинской томографии (рентгеновская, ЯMP, PET, SPECT), а также по промышленной 
и физической томографии. Ведется постоянное пополнение данных по текущим конференциям, активно работающим группам по вычислительной томографии и обратным задачам, специалистам в томографии и основным книгам по данной тематике. Представлен ресурс с библиографической базой данных группы Вычислительной томографии Института теоретической и прикладной механики им. С. А. Христиановича СО РАН, содержащей около 6000 записей по различным направлениям теории обратных задач и их приложений: томография, вэйвлет-анализ, методы регуляризации, биоинформатика, обработка сигналов и изображений.

Очевидно, что при накоплении объемов информации потребуется бо́льшая специализация ресурсов. Поэтому в системе MathTree предусмотрена возможность создания дополнительных подразделов в соответствии с рубрикатором MSC.

Как мы отмечали выше, основная цель создания системы MathTree - упорядочение существующих и вновь создаваемых математических ресурсов по указанными выше математическим направлениям и типам. Наряду с этим в системе предусмотрена возможность создания новых ресурсов, которая обусловлена не только необходимостью пополнения перечисленных выше разделов и дополнительного сервиса для пользователей, но и потребностью в создании отдельных разделов, которые представляют интерес для широкого круга лиц, интересующихся математикой и историей математики. Об одном таком разделе будет рассказано ниже.

В современном мире, помимо проблемы распространения информации, остро стоит проблема сохранения знаний человечества и его наследия. В связи с развитием современных технологий и созданием новых способов хранения данных во всем мире ведется работа по сохранению источников информации (книги, музыкальные произведения, фильмы и т.д.) в цифровом формате, в том числе такая работа ведется по математическим журналам и книгам. В частности, с 2002 года по инициативе ряда математических обществ, издательства "Шпрингер" и четырех библиотек: библиотеки Корнельского университета (Нью-Йорк), государственной университетской библиотеки Гёттингена, библиотеки университета Чинхуа (Пекин), математической библиотеки Opсе (Париж), в рамках проекта EMANI [14] ведется работа по представлению в цифровом формате полных текстов статей из математических журналов, опубликованных в 18-20 веках. В настоящее время созданы и постоянно пополняются цифровые библиотеки математических книг, вышедших в 17-20 веках; см., например, коллекцию цифровых математических книг Национальной библиотеки Франции [15] или историческую коллекцию математики Мичиганского университета [16]. Дополнительные сведения о цифровых математических библиотеках заинтересованный читатель найдет, обратившись к проекту “Всемирная цифровая библиотека математики" [17], инициатором которого выступил комитет по электронной информации и коммуникациям при Международном математическом союзе.

Полнотекстовые коллекции журналов и книг в цифровом формате создаются и в России, при этом особое внимание уделяется старопечатным и редким книгам (см., например, [18]-[20]). В настоящее время создан ряд цифровых коллекций математических журналов и книг. В частности, в упомянутой выше информационной системе Math-Net.Ru ключевым является раздел “жсурналь”. 
В настоящее время в нем представлена информация о публикациях за последние 10-30 лет в 17 журналах. С целью охватить все номера журналов, работы в этом направлении продолжаются. Уже сейчас пользователь может познакомиться с полными текстами статей первого выпуска журнала "Математический сборник", вышедшего в 1866 году. В рамках российско-германского проекта "Российская электронная математическая библиотека" также ведутся работы по представлению русскоязычных журналов в электронном виде [21]. В 2003 году в библиотеке Математического института им. В. А. Стеклова РАН начались работы по созданию полнотекстовой базы, отражающей фонды библиотеки [22].

К сожалению, число коллекций старинных математических книг, хранящихся в российских библиотеках, особенно на русском языке, в цифровом формате ничтожно мало. Среди существующих следует отметить Интернет-библиотеку Московского центра непрерывного математического образования [23], в которой, например, представлена уникальная книга Л.Ф. Магницкого “Арифметика", изданная в 1703 году и хранящаяся в отделе редких книг и рукописей научной библиотеки Московского государственного университета.

Учитывая существующий интерес к таким коллекциям, с целью сохранения и обеспечения доступа к ним максимально большого круга читателей в системе MathTree был создан раздел "Старинные книги по математике". Основу этой коллекции составляют фонды библиотек научно-исследовательских институтов Сибирского отделения Российской академии наук. В настоящее время в этом разделе представлены полные тексты нескольких книг, хранящихся в библиотеке Института математики им. С. Л. Соболева СО РАН:

Д.А. Граве, Об интегрировании частных дифференциалъных уравнений первого порядка, Диссертация на степень магистра чистой математики, Императорская Академия наук, Санкт-Петербург, 1889;

Н. И. Лобачевский, Алгебра или вычисление конечных, Казанский университет, Казань, 1834;

А. А. Марков, Доказательство трансцендентности чисел е и ность квадратуры круга), Императорская Академия наук, Санкт-Петербург, 1883 ;

А. А. Марков, Исчисление конечных разностей, Императорская Академия наук, Санкт-Петербург, 1889, 1891;

J. Bernoulli, Nouvelles pensées sur le système de $M$. Descartes, et la manière d'en déduire les orbites $\&$ les aphélies des planètes, Paris, 1730.

Помимо текстов для каждой книги дается краткое описание представленного материала и ссылки на электронные ресурсы, посвященные авторам книг.

\section{4. Возможности системы}

Кратко описав предлагаемую в системе MathTree информацию, ниже мы хотели бы остановиться на ее возможностях.

Поскольку наполнение системы происходит по направлениям математики, причем степень специализации может быть достаточно высокой, то каждый раздел курируют специалисты в соответствующей области математики, работающие в научно-исследовательских учреждения Российской академии наук: 
- Институт математики им. С. Л. Соболева СО РАН;

- Институт систем информатики им. А. П. Ершова СО РАН;

- Институт вычислительной математики и математической геофизики CO PAH;

- Институт вычислительных технологий СО РАН;

- Институт теоретической и прикладной механики им. С. А. Христиановича $\mathrm{CO} \mathrm{PAH}$;

- Институт автоматики и электрометрии СО РАН;

- Институт гидродинамика им. М. А. Лаврентьева СО РАН;

- Институт автоматики и процессов управления ДВО РАН.

Привлечение экспертов и узких специалистов в качестве создателей и редакторов разделов, а также подразделов системы MathTree способствует высокой степени структурированности и достоверности предлагаемой математической информации.

При наполнении системы по каждому ресурсу вводятся следующие данные: тип ресурса; язык, на котором представлено содержание ресурса; адрес ресурса; название ресурса; краткое описание содержания ресурса; ключевые слова. Поскольку информация в системе представлена на русском и английском языках, то название ресурса, его описание и ключевые слова вносятся на обоих языках. В результате по каждому ресурсу система предлагает пользователю следующую информацию.

- Раздел. Название раздела с указанием всех подразделов генерируется системой автоматически, в зависимости от того, в каком разделе и на каком уровне находится пользователь.

- Адрес. Указан web-адрес ресурса.

- Ресурс. Указано название ресурса на соответствующем языке.

- Описание. Представлено краткое описание содержания ресурса на соответствующем языке. В качестве дополнительной информации в описании могут содержаться ссылки на вспомогательные ресурсы.

- Категория. Указан тип ресурса: журналы, конференции, персоны, публикации, библиотеки, организации, научные сообщества, программное обеспечение, научные школы, семинары, другое.

- Индекс в классификаторе. Генерируется системой автоматически в соответствии с рубрикатором MSC в зависимости от того, в каком разделе и на каком уровне находится пользователь.

- Дата последнего отклика ресурса. Указана дата внесения информации о ресурсе в систему, дата генерируется системой автоматически.

- Язык. Указан язык, на котором представлено содержание ресурса.

Эта дополнительная информация помогает пользователю получить предварительные сведения о ресурсе и принять решение о необходимости обращения к самому ресурсу. Информация, которая вносится по каждому ресурсу, позволяет пользователю также осуществлять поиск нужных сведений по отдельным словам и фразам, при этом он проводится не только по ключевым словам, внесенным при описании ресурса, но также по фразам, содержащимся в названии и описании содержания ресурса. Помимо этого в системе предусмотрены следующие дополнительные возможности: 
- навигация по связанным ресурсам;

- возможность автоматически добавлять информацию из других баз данных;

- возможность добавлять информацию пользователями системы;

- разграничение права доступа между пользователями.

Последние три из указанных возможностей связаны с процессом наполнения системы, которое проводится как вручную, так и автоматически. Ручной способ является самым трудоемким, весьма затратным по времени, но самым надежным в отношении адекватности предлагаемой информации, чего нельзя сказать об автоматическом. В настоящее время автоматический способ наполнения системы с использованием существующих баз данных осуществляется только по публикациям в журналах. При этом сгенерированная системой информация проверяется на предмет соответствия специалистами в соответствующей области. Говоря о наполнении системы вручную, остановимся на группах пользователей системы.

Первую группу составляют администраторы данных. Как отмечалось выше, в нее входят специалисты в соответствующей области математики, которые осуществляют наполнение системы (создание, редактирование, удаление ресурсов) и контролируют адекватность предоставляемой информации. Бо́льшая часть информации вносится именно этой группой пользователей.

Вторую группу составляют зарегистрированные пользователи, достаточно компетентные в своей области, которые также имеют возможность вносить информацию в систему. Предоставляемая ими информация, при необходимости, редактируется администраторами. С развитием системы и распространением информации о MathTree эта группа пользователей начинает играть более заметную роль.

Третью группу составляют все остальные пользователи, на которых были ориентированы создание системы MathTree и ее возможности по поиску нужной информации.

В процессе дальнейшего развития MathTreе планируется расширить возможности системы, а именно, предполагается: классификация ресурсов с использованием УДК (универсальная десятичная классификация) и рассылка сообщений пользователям системы (по подписке) с информацией о добавленных pecypcax.

\section{5. Современное состояние системы}

В настоящее время в системе представлена информация о более чем 4000 ресурсах. На момент написания статьи по основным математическим разделам степень наполнения была следующей: алгебра и логика - 260 ресурсов; анализ - 263 ресурса; геометрия и топология - 159 ресурсов; дифференциальные, интегральные и разностные уравнения - 986 ресурсов; теория вероятностей и математическая статистика - 207 ресурсов; математическое программирование и Computer Science - 1639 ресурсов; приложения и другое - 688 ресурсов.

Согласно классификации по типам, представленные ресурсы охватывают 162 журнала, 185 конференций и 349 страниц с информацией о биографиях и деятельности ученых. Как правило, ресурсы по перечисленным типам составляют базы данных математических обществ и организаций, поэтому в 
системе MathTree пользователь найдет также ссылки на эти базы данных. На момент написания статьи в системе была представлена информация о 2340 публикациях, что составляет более половины ресурсов MathTree. Это связано с использованием указанного выше автоматического способа наполнения системы. Ресурсы, систематизированные по типу “организации”, охватывают 471 организацию, научное или образовательное подразделение, при этом в системе содержится информация о 87 научных сообществах и группах. Как мы отмечали выше, к сожалению, не у каждой ведущей научной школы РФ в настоящее время имеется электронный ресурс, посвященный ее истории и направлениям исследований. Именно этим объясняется малое количество представленных ссылок, пока 42. В системе MathTreе представлена информация о 147 ресурсах, посвященных программному обеспечению, при этом, естественно, бо́льшая их часть содержится в разделе "Математическое программирование и Computer Science". Классификация по типам "библиотеки" и "семинарь" была введена недавно, и пока в системе MathTreе представлено 86 ресурсов, относящихся к этим типам, 431 математический ресурс отнесен к типу "другое".

За время существования системы MathTree увеличилось не только число пользователей, у которых предлагаемая информация вызывает интерес, но и тех, кто участвует в наполнении системы. Это позволяет надеяться, что система MathTree будет успешно развиваться в дальнейшем. Авторы статьи приглашают к сотрудничеству всех заинтересованных читателей и будут признательны за конструктивные предложения и замечания. Комментарии следует направлять ученому секретарю проекта к.ф.-м.н. О. А. Клименко по адресу: klimenko@mathtree.ru.

\section{Список цитируемых Интернет-ресурсов и литературы}

[1] Справочно-информационная система MathTree, http://www.mathtree.ru.

[2] Ю. Л. Ершов, О. А. Клименко, И. И. Матвеева, Л. Р. Рабинович, В. Э. Филиппов, М.Я. Филиппова, "Древовидный каталог математических интернет-ресурсов", Информачионные ресурсы России, 2006, № 1, 5-8.

[3] А. С. Аджиев, "WEB-ресурсы для российских математиков", Информационнъе ресурсы России (вкладыш), 1-20, №6.

[4] Google Scholar, http://scholar.google.com.

[5] International Mathematical Union, http://www mathunion.org.

[6] American Mathematical Society, http://www.ams.org.

[7] European Mathematical Information Service, http://www.emis.de.

[8] Математические ссылки. Санкт-Петербургское математическое общество, http://www. mathsoc.spb.ru/links-fr.html.

[9] Mathematics Web Sites. Pennsylvania State University, http://www . math.psu.edu/MathLists.

[10] Научный портал механико-математического факультета МГУ, http://shade.lcm.msu.ru:8080/content_root/links.jsp.

[11] Mathematics e-Resources. Technical Library. Sandia National Laboratories, http://infoserve.sandia.gov/electronic/mathdb.html.

[12] Математика в Интернет. Библиотека по естественным наукам РАН, http://www. benran.ru/E_n/mathint.htm.

[13] Общероссийский математический портал Math-Net.Ru, http://www . mathnet.ru. 
[14] Electronic Mathematics Archives Network Initiative, http://www.emani.org.

[15] Livres Numérisés Mathématiques, http://math-doc.ujf-grenoble.fr/LiNuM/LiNuM_en.html.

[16] The University of Michigan Historical Mathematics Collection, http://www.hti.umich.edu/u/umhistmath.

[17] World Digital Mathematics Library, http://www.wdml.org.

[18] С. К. Куранов, А. В. Скоробогатов, В. Д. Соловьев, Е. Л. Столов, “Электронная коллекция отдела рукописей и редких книг Казанского госуниверситета", Информачионное обеспечение науки: Новые технологии, Сборник научных трудов, БEH PAH, M., 2001, 141-145; http://www . benran.ru/Magazin/cgi-bin/Sb_01/pr01.exe?! 26.

[19] С. Р. Баженов, В. Н. Алексеев, А. Ю. Бородихин, Е. И. Дергачева-Скоп, А. В. Шабанов, "Создание цифровых коллекций редких книг и рукописей из сибирских хранилищ”, Информачионное обеспечение науки: Новые технологии, Сборник научных трудов, БЕН РАН, М., 2001, 146-148.

[20] А.Ц. Масевич, Е.А. Савельева, А. К. Багажков, В.П. Захаров, “Электронная библиотека старопечатных книг в Библиотеке Российской академии наук”, Электронные библиотеки, 5:1 (2002); http://www.elbib.ru/index.phtml?page=elbib/rus/journal/2002/part1/MZBZ.

[21] Б.Л. Крайнова, Е.И. Глухова, Российские математические электронные ресурсы. Совместный российско-германский проект "Российская электронная математическая библиотека", Труды тринадцатой международной конференции "Крым 2006": "Библиотеки и информационные ресурсы в современном мире науки, культуры, образования и бизнеса", 2006; http://www .gpntb.ru/win/inter-events/crimea2006/disk2/160.pdf.

[22] К. П. Погорелко, "Вопросы создания полнотекстовой базы данных в библиотеке Математического института им. В. А. Стеклова РАН", Информационное обеспечение науки: Новые технологии, Сб. науч. трудов, БЕН РАН, М., 2005, 270-274.

[23] Интернет-библиотека Московского центра непрерывного математического образования, http://ilib.mccme.ru.

Ю. Л. Ершов (Yu. L. Ershov)

Институт математики им. С. Л. Соболева СО РАН

E-mail: ershov@math.nsc.ru

\section{О. А. Клименко (О. А. Klimenko)}

Институт вычислительных технологий СО РАН

E-mail: klimenko@ict.nsc.ru

\section{И. И. Матвеева (I. I. Matveeva)}

Институт математики им. С. Л. Соболева СО РАН

E-mail: matveeva@math.nsc.ru

\section{В. В. Пикалов (V. V. Pikalov)}

Институт теоретической и прикладной механики СО РАН

E-mail: pickalov@itam.nsc.ru
Поступила в редакцию 22.06.2007 